\title{
Death of a Severe Underweight Infant with Pulmonary Interstitial Glycogenosis
}

\author{
Seon Jung Jang ${ }^{2}$, \\ Kyung Ryoul Kim, \\ Hyoung Joong Kim', \\ Minjung Kim ${ }^{1}$ \\ ${ }^{1}$ Division of Forensic Medicine, \\ National Forensic Service, Seoul, \\ Korea \\ ${ }^{2}$ Department of Forensic Medicine, \\ Korea University College of \\ Medicine, Seoul, Korea
}

Received : October 23, 2012

Revised : November 17, 2012

Accepted : November 20, 2012

Corresponding Author : Minjung Kim Division of Forensic Medicine, National Forensic Service, 331-1 Sinwol 7dong, Yangchoen-gu, Seoul, 158-707, Korea.

TEL : +82-2-2600-4632

FAX : +82-2-2600-4649

E-mail : lillu79@korea.kr

\begin{abstract}
Pulmonary interstitial glycogenosis (PIG) is a very rare interstitial lung disease in infants. It is poorly understood, but its pathological features are distinct; they include uniform alveolar septal thickening, caused by a group of oval to spindle-shaped cells containing abundant glycogen, without apparent inflammation or fibrosis. PIG is usually associated with a good prognosis. However, in the present report, we describe the case of a 5-month-old male infant who died due to PIG; he was severely underweight and not administered proper treatment or care. The pathology of PIG was determined following a medico-legal autopsy.
\end{abstract}

Key words : infant, pulmonary interstitial glycogenosis, underweight, death

\section{Introduction}

Interstitial lung disease (ILD) in infants and children is a very rare cause of respiratory distress during the neonatal period and consists of broader spectrum of disorders than in adults. ${ }^{1,2)}$ This is certainly linked to the fact that the disease occurs in the context of lung growth at the various stages of alveolar development and maturation, with each of these stages being regulated by specific cascades of events. ${ }^{3 !}$

One of ILD, Pulmonary Interstitial Glycogenosis (PIG) is newly reported disease entity in 2002 by Canakis and his collegues. ${ }^{4}$ It is also called infantile cellular interstitial pneumonia or cellular interstitial pneumonitis of infancy, and is a poorly understood entity of the infantile lung disease. Here we report an autopsy case of PIG with a severely underweight, 5month-old male infant reviewing the relevant literatures.

\section{Case Report}

A 5-month-old male infant was born to a 23-year-old gravida 2, para 1 mother at about 38 weeks gestation by normal spontaneous vaginal delivery. The infant was at birth stained with meconium $(3+)$, with APGAR scores of 7 and 9 at 1 and 5 minutes, 
respectively, and had a birth weight of 3,858 g. He was diagnosed as meconium aspiration syndrome and admitted to the neonatal intensive care unit. The complete blood cell count study revealed a white cell count of 24,570 cells $/ \mathrm{mL}$ and a total bilirubin level of $3.32 \mathrm{mg} / \mathrm{dL}$ in serum. He was treated by the antibiotics, ampicillin and gentamicin for 4 days. Then the biologic study for the blood and urine was negative and chest radiography exams showed no remarkable abnormality, and he was discharged. After 5 months, he suddenly collapsed at home and was dead on arrival at the hospital. According to his mother, he had a fever, lethargy, and poor appetite for 2 days before death. His parents did not take him to

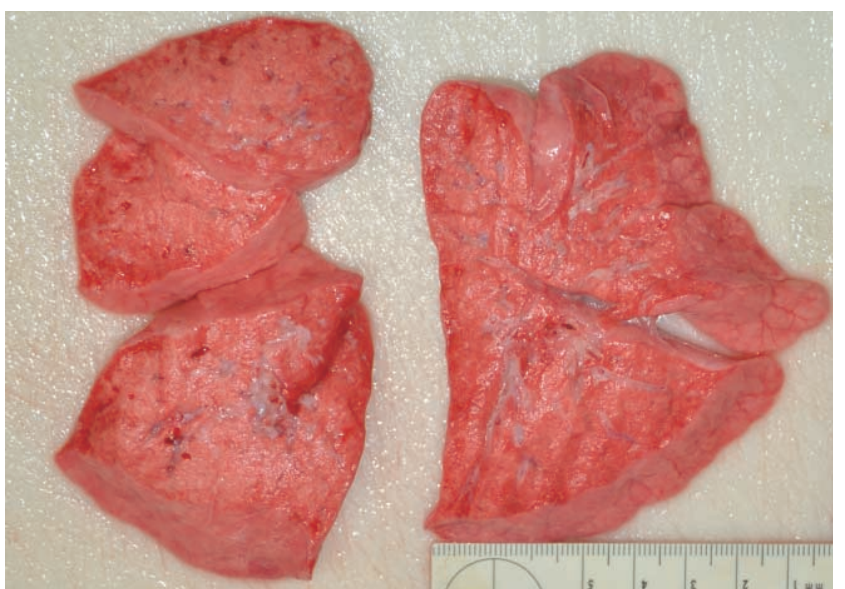

Fig. 1. The both lungs were pale salmon in color and spongy like appearance in cross section.

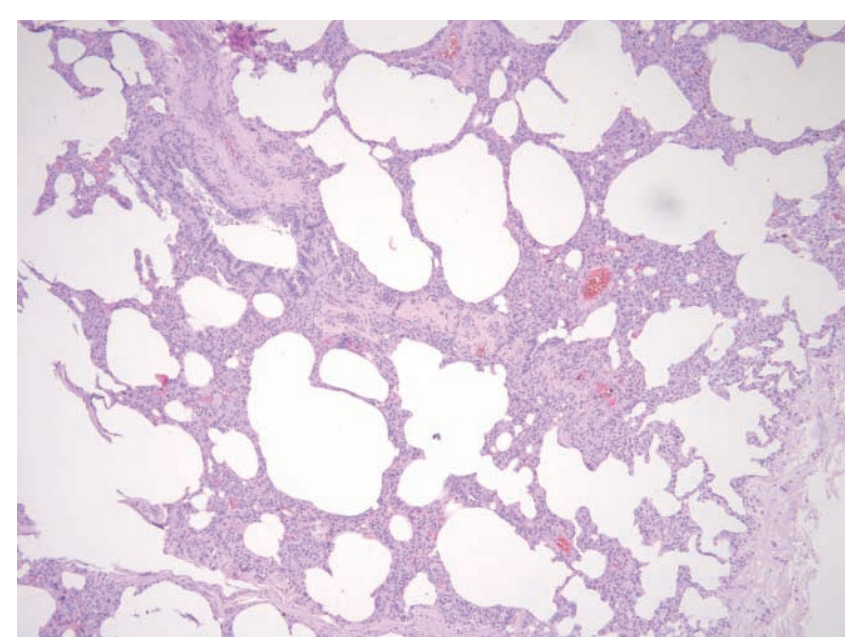

Fig. 2. Diffuse interstitial thickening without alveolar epithelial hyperplasia or protenaceous material in alveolar space $(H \& E, \times$ 100). the hospital and he had never been vaccinated for financial reasons for five months. His parents thought that he died from the hot temperature of the rooftop house room they live in. The incident happened in one hot summer day and the police investigators found the room temperature was $33^{\circ} \mathrm{C}$ at $14 \mathrm{o}^{\prime}$ clock. The infant's older brother, 3-year-old, was healthy without any disease or malnutrition.

The external examination revealed that the infant was severely underweight. His body weight, 4,105 g was less than the 3 percentile with the stature at the 25 percentile. The orbits were sunken and the abdomen was markedly thin. The skin turgor was obviously decreased. Several scratches were noted on his face, which might be inflicted from the uncared finger nails of the infant. On internal examination, the both lungs were pale salmon in color and the crosssection of those showed kind of sponge-like consistency (Fig. 1). There was no evidence of disease or malformation in other organs. Microscopically, both lungs showed diffuse interstitial thickening without apparent hyperplasia of alveolar epithelial cells or marked accumulation of cells or proteinaceous material within the alveolar spaces. The interalveolar septae were seen to be generally increased with ovalto spindle-shaped cells with pale cytoplasm, indistinct cell membrane, and oval, bland nuclei. There was no distinct inflammatory cell infiltration or fibrosis. The

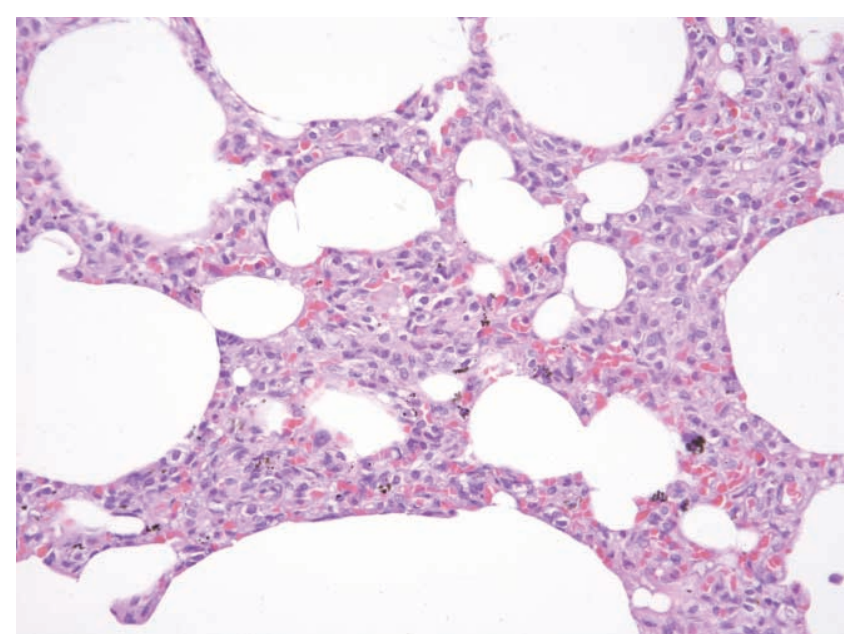

Fig. 3. Marked increased interstitial mesenchymal cells showing vacuolated cytoplasm and oval and bland nuclei $(H \& E, \times 400)$. 
periodic acid-Schiff (PAS) reacted positively with diastase sensitive material within the cytoplasm of the oval to spindle-shaped cells, possibly indicating the presence of glycogen. The alveolar space was irregularly expanded (Figs. 2-4). No drugs, poisonous agents or chemicals were detected in the blood and the gastric tissue. The urea nitrogen, creatinine, sodium and chloride level in the vitreous humor were $79.2 \mathrm{mg} / \mathrm{dL}, 0.4 \mathrm{mg} / \mathrm{dL}, 159 \mathrm{mEq} / \mathrm{L}$, $149 \mathrm{mEq} / \mathrm{L}$, respectively, indicating a severe dehydration. The bacterial culture study for the blood was negative.

The main medicolegal autopsy findings were interstitial lung disease featuring PIG, dehydration and very low body weight. We interpreted the infant's death that he died of both untreated interstitial lung disease, PIG and dehydration. We guessed that both the internal factor, PIG and external one, the parent's inappropriate care contributed to the infant death.

\section{Discussion}

Pulmonary interstitial glycogenosis is one of interstitial lung disease first proposed by Canakis et al. ${ }^{4)}$ in 2002 presenting tachypnea, hypoxemia and diffuse interstitial infiltrates with over inflated lungs on chest radiographs in the first month of life. In biopsied specimen of 7 infants, it showed expansion of the interstitium by spindle-shaped cells containing periodic acid-positive diastase labile material

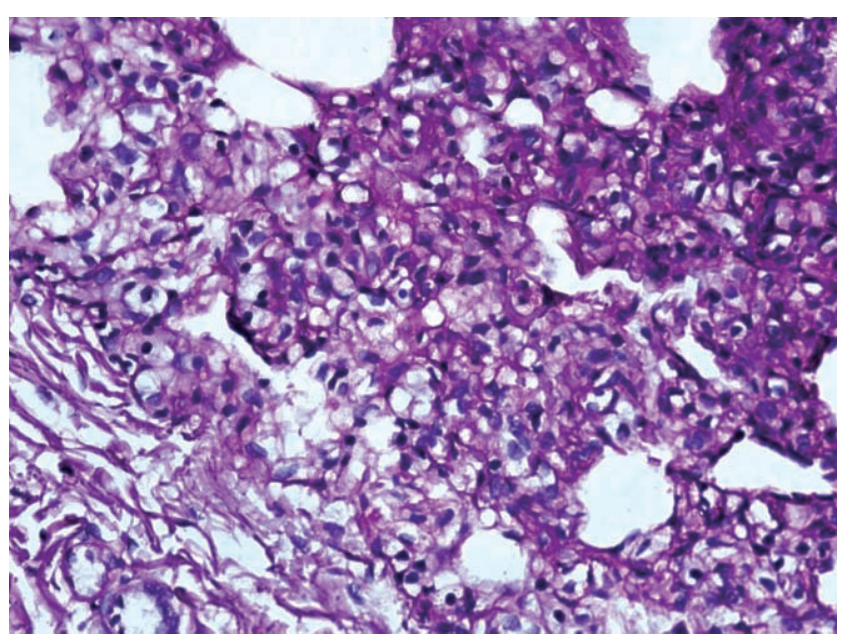

Fig. 4. The cytoplasm of interstitial cells displaying weak PAS positive diastase sensitive material (PAS, $\times 400$ ). consistent with glycogen. However the preservation of glycogen varied between cases because of the use of aqueous fixative. Inflammatory cells are rarely found in this disease. The function of interstitial cell is unclear and the etiology of the disease is not known yet. The dysmaturity of interstitial cells found in PIG in contrast to UIP or DIP characterized inflammatory process. Onland suggested a disturbance in a developmental pathway with genetic component as etiology describing PIG in monozycotic twins. ${ }^{51}$

PIG is a vastly under-recognized and underreported histologic pattern, and is a relatively common feature of biopsies for diffuse lung disease in infants less than six months of age, particularly those with other superimposed forms of lung injury, for example, alveolar growth abnormality, pulmonary arteriopathy, meconium aspiration, compressed lung adjacent to congenital lung malformations, and others. ${ }^{2)}$

Although PIG has a favorable prognosis treated with oxygen supplement, steroid treatment or spontaneous resolution, ${ }^{6}$ some of them showed negative outcome. Radman et $\mathrm{al}^{7 /}$ reported two cases of respiratory distress symptom with persistent pulmonary hypertension and congenital heart disease. Canakis et $\mathrm{al}^{4 /}$ reported a fetal case with PIG and death due to cor pulmonale. King et $\mathrm{al}^{8}$ reported a case of PIG with abnormal lung growth and death due to extreme lung dysmaturity. They mean that the prognosis of PIG is dependent of co-morbidity.

In our case, the neonate is a full term baby with the history of meconium aspiration at birth without any familial history of respiratory or heart disease. While he had respiratory difficulties in post-term due to meconium aspiration after birth, he was improved and discharged with administration of antibiotics and supportive care. But thereafter he had not been supported with appropriate medical treatment and had been even neglected by his parents. It is considered that these risk factors made him severely underweight, dehydrated and dead under the influence of the infant pathologic lung state, PIG. 


\section{References}

1. Deterding RR. Infants and young children with children's interstitial lung disease. Pediatr Allergy Immunol Pulmonol 2010;23:25-31.

2. Dishop MK. Paediatric interstitial lung disease: classification and definitions. Paediatr Respir Rev 2011;12:230-7.

3. Clement A, Eber E. Interstitial lung diseases in infants and children. Eur Respir J 2008;31:658-66.

4. Canakis AM, Cutz E, Manson D, O'Brodovich $\mathrm{H}$. Pulmonary interstitial glycogenosis: a new variant of neonatal interstitial lung disease. Am J Respir Crit Care Med 2002;165:1557-65.
5. Onland W, Molenaar JJ, Leguit RJ, et al. Pulmonary interstitial glycogenosis in identical twins. Pediatr Pulmonol 2005;40:362-6.

6. Deutsch GH, Young LR. Histologic resolution of pulmonary interstitial glycogenosis. Pediatr Dev Pathol 2009;12:475-80.

7. Radman MR, Goldhoff $\mathrm{P}$, Jones KD, et al. Pulmonary interstitial glycogenosis: an unrecognized etiology of persistent pulmonary hypertension of the newborn in congenital heart disease? Pediatr Cardiol 2012.

8. King BA, Boyd JT, Kingma PS. Pulmonary maturational arrest and death in a patient with pulmonary interstitial glycogenosis. Pediatr Pulmonol 2011;46:1142-5. 